\title{
Re: The Clinical and Urodynamic Results of Percutaneous Posterior Tibial Nerve Stimulation on Neurogenic Detrusor Overactivity in Patients with Parkinson's Disease
}

\author{
Şahin Kabay1, Sibel Canbaz Kabay2, Mustafa Çetiner3, Emine Mestan3, Mehmet Sevim3, \\ Selahattin Ayas2, Hilmi Özden4, Handan Özıșık Karaman5 \\ 1 Dumlupınar University Faculty of Medicine, Department of Urology, Kütahya, Turkey \\ 2Dumlupınar University Faculty of Medicine, Department of Neurology, Kütahya, Turkey \\ ${ }^{3}$ Dumlupınar University Faculty of Medicine, Department of Urology, Kütahya, Turkey \\ 4Eskişehir Osmangazi University Faculty of Medicine, Department of Anatomy, Eskişehir, Turkey \\ 5 Çanakkale 18 Mart University Faculty of Medicine, Department of Neurology, Çanakkale, Turkey
}

Urology 2015. pii: S0090-4295(15)00927-9. doi: 10.1016/j.urology.2015.09.026. [Epub ahead of print]

\section{EDITORIAL COMMENT}

Parkinson's disease (PD) is a progressive disease displaying itself through dopaminergic neuron loss. Lower urinary tract symptoms (LUTS) observed in this patient group are a result of autonomic disorders. The most frequently observed urodynamic abnormalities are neurogenic detrusor overactivity (ND0), hyporeflexia or areflexia, decreased capacity and abnormalities of external sphincter function. Generally in this patient group, the first treatment approach is pharmacotherapy. In resistant patients, botulinum toxin (BTX) injections are a treatment approach. Kabay et al. designed their study based on successful results of posterior tibial nerve stimulation (PTNS), a minimally invasive neuromodulation technique, in neurogenic and non-neurogenic LUTS patient groups resistant to medical treatment. The study included 47 patients with PD diagnosis and NDO. All patients were administered 12 weeks of percutaneous PTNS. Voiding diary, urodynamic studies, and questionnaires (ICIQ-SF, OAB-V8, OAB-qSF) were completed by all patients before and after treatment. The results of the study of 26 males and 21 females with a mean age of $61 \pm 8.3$ years identified $7.3 \pm 3.8$ years of PD and $3.6 \pm 2.4$ years of LUTS. A significant improvement was observed in all urodynamic variables $(p<0.001)$. Similarly, there were significant improvements in voiding diary parameters, ICIO-SF, OAB-V8 and $0 A B-q$ in patients after treatment ( $p<0.001)$. The researchers concluded that these findings showed that PTNS treatment provided improvement of LUTS and urodynamic parameters in PD patients. PTNS has become very popular in recent times and is a treatment approach frequently seen in current literature. I am of the opinion that sharing the results of this treatment applied to a selective patient group like PD makes this study valuable. 